\title{
Research of Flexible Visual Experiment Platform for Communication Principle Teaching
}

\author{
Yihuai Yang \\ School of Automatic Control and Mechanical Engineering, Kunming University, 650214, China \\ email: yyh_td@126.com
}

Keywords: Matlab/Simulink, Visual Simulation, Teaching Method, Amplitude Modulation, Sample, FM Radio

\begin{abstract}
Traditional hardware experimental platforms are no long adapt to the fast updating knowledge of communication technologies. In this paper, we studied the characteristic of experiments of communication principle, analyzed the drawbacks of existing hardware experiments, and proposed that traditional hardware experiments should be assisted by using Matlab/Simulink software, due to the fact that this advanced software can be a very useful teaching and researching assistant aid tool for traditional hardware experimental platforms. We give three typical examples which are hard to understand for students when using traditional teaching methods. We also give the simulation results in vivid and real-time figures. The analysis of the examples shows that applying Matlab/Simulink to set up flexible visual experiment platform for communication principle teaching is feasible and efficient.
\end{abstract}

\section{Introduction}

Communication Principle is one of the most important course in specialized elementary course for Telecommunication, Electronic Information, Automatic Control and Electricity in universities. If students and researchers could master the technologies of communication, they could get a deep understanding of relative courses, such as signal and systems, mobile and wireless communications, antenna technologies ect., in an much easier and better way. However, due to the fact that there are so many complicate concepts, modulation techniques and various systems in this course, it is difficult and hard to learn this course. How to make this course easy to learn is always a hard project that teachers are studying and researching. The transfer of traditional teaching method 'teachers in dominant position' to 'students in dominant position' could initiate students' active roles in learning courses.

Compared to other languages and method, Matlab/Simulink has a lot of advantages, for example [1]:

- A very large (and growing) database of built-in algorithms for image processing and computer vision applications

- Vectorized operations.

- The graphical output is optimized for interaction.

- Matlab’s functionality can be greatly expanded by the addition of toolboxes. etc.

And most importantly, it is very easy to learn and quick apply to practice problems. The application of Matlab/Simulation in the teaching of Communication Principle, teachers and students could avoid many useless and complicate mathematical derivations. And in this way, students selfdirected learning can play the vital role in the process of teaching.

\section{The Necessity of Reforming Communication Experimental Platform}

The reformation of communication experimental platform is crucial, due to the fact that traditional communication experiments are mostly replication experiment base on hardware and has many drawbacks, such as: 
- Outdated equipment can not meet the request of the fast developing modern telecommunication technologies, and also can not match updated lecture materials.

- The content of experiments is fixed because of the equipment limit, as a result, students can not do flexible experiments by doing second development.

- The construction and maintenance of hardware of the laboratory need large amount of human and financial resources.

- Different hardware based experiments are relatively independently, so it is hard for students to get systematic knowledge.

As one of the most advanced and popular program design languages, Matlab\Simulink not only can do all the experiments that could be done on hardware experiment platform, but also could conduct some updated and innovative experiments, even some research work, which could not be done on the traditional hardware experiment platform.

\section{Typical Examples}

In this section, we give some typical Matlab/Simulink based examples, which include some very important concepts and must be learn knowledge in the course of Communication Principle.

\subsection{FM Stereo Model}

In telecommunications and signal processing, frequency modulation (FM) is the encoding of the sound or other information that is required to be placed in a carrier wave by varying the instantaneous frequency of the wave [3]. FM broadcasting is a method of radio broadcasting using frequency modulation (FM) technology. Invented in 1933 by American engineer Edwin Armstrong, wide-band FM is used worldwide to provide high-fidelity sound over broadcast radio[4]. FM stereo demodulation is also called stereo decoder, in this example, the digital FM stereo decoder uses two BPF and one LPF, together with a summator and a subtractor. A low distortion output is obtained without the use of a phase locked loop for the regeneration of the subcarrier signal.

The principle block diagram of the FM Stereo Model is shown in Figure 1. And in Figure 2, we use Matlab/Simulink modeling and simulation study the FM stereo system. The visual simulation results are shown in Figure 3. From Figure 3, we can see that at the receiver, the signal wave has a little bit distortion compared with the wave at the transmitter, this is because of the white noise.

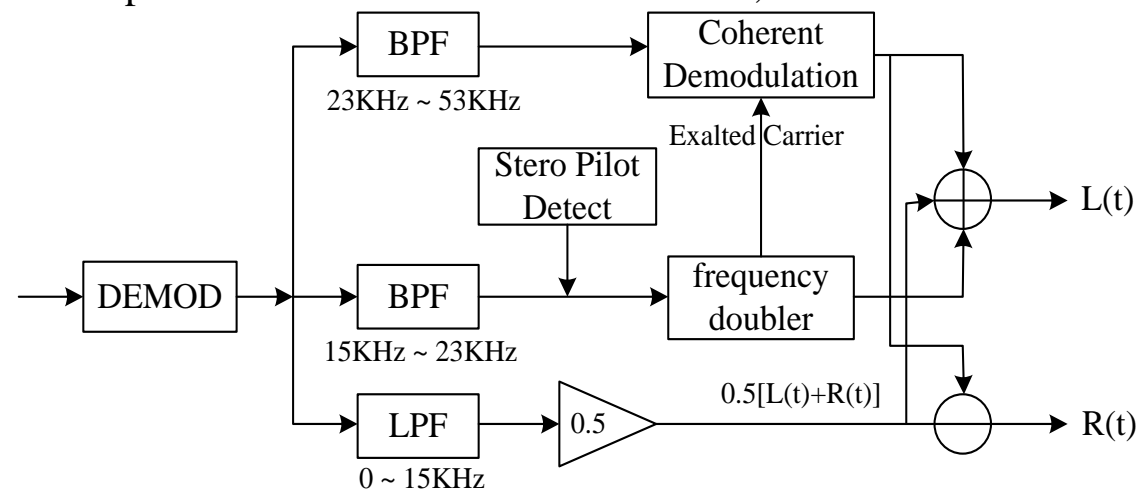

Figure 1. Principle block-diagram of the FM stereo model 


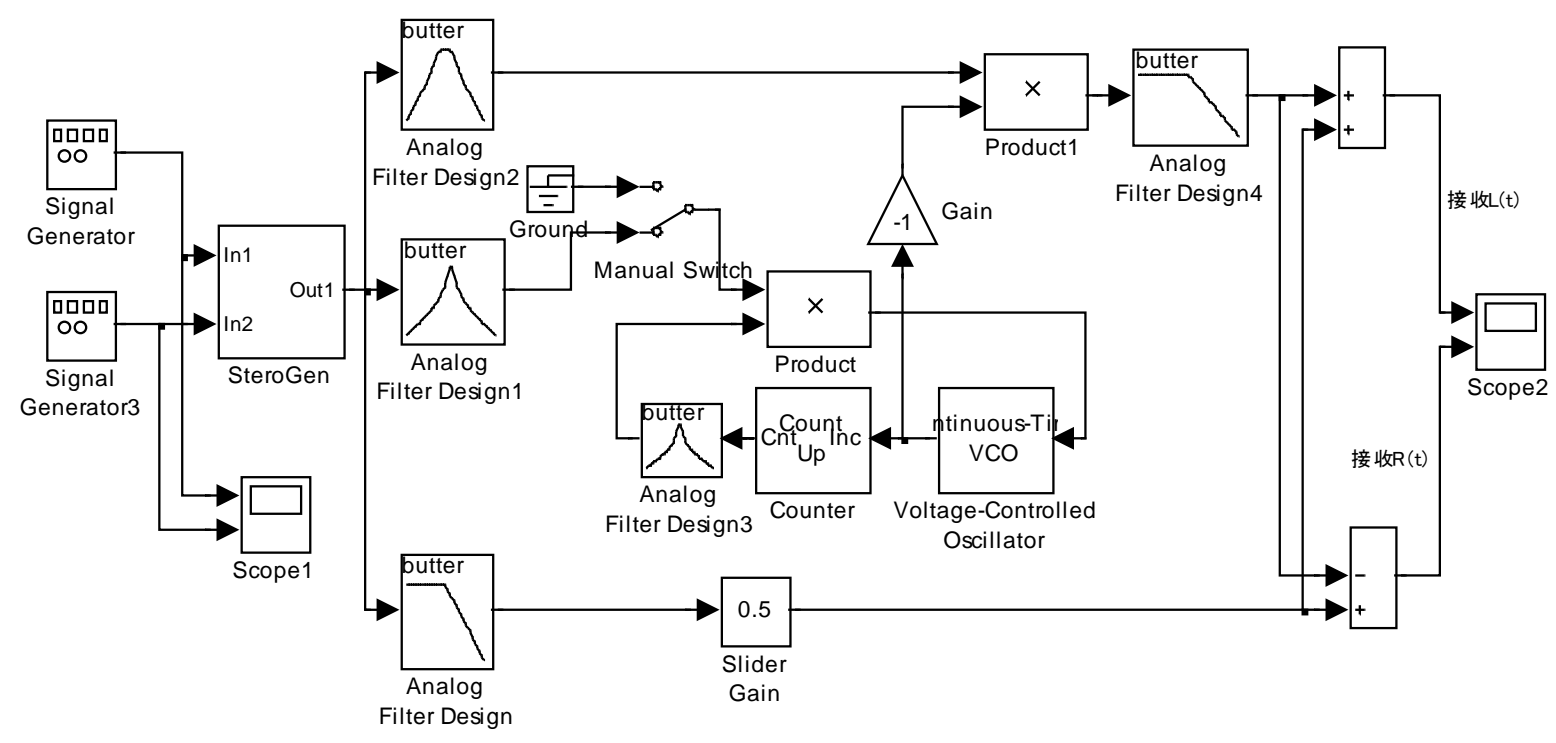

Figure 2. FM Stereo Decoding Test Model
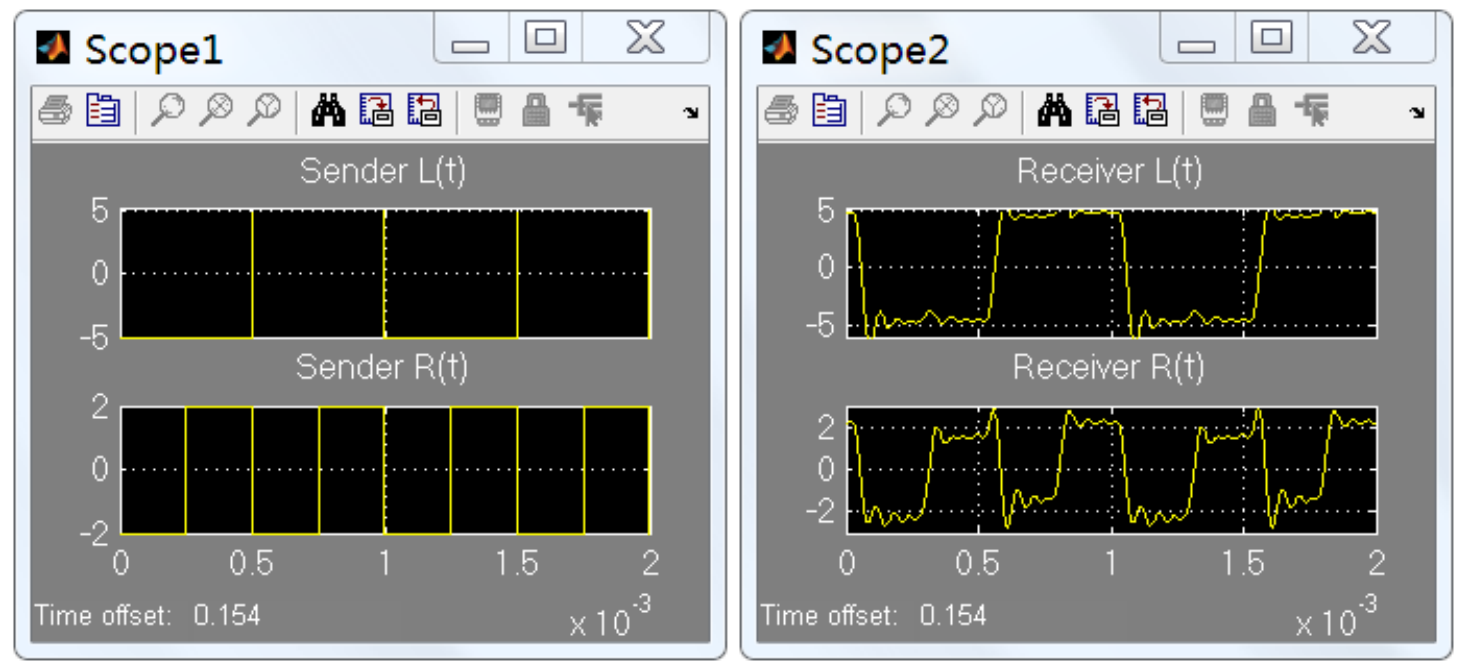

Figure 3. Waveform of Transmitter- and Receiver-side waveform

\subsection{Pulse Amplitude Modulation/Demodulation Model}

Pulse Amplitude Modulation (PAM) [5], is one of the simplest form of signal modulation, which transmitting of information by varying the the amplitude (voltageor power levels) of a series of signal pulse in a regularly timed sequence. The PAM Modulation \& Demodulation Sample and Hold Model is given in Figure 4 [6]. and the results are shown in the Figure 5.

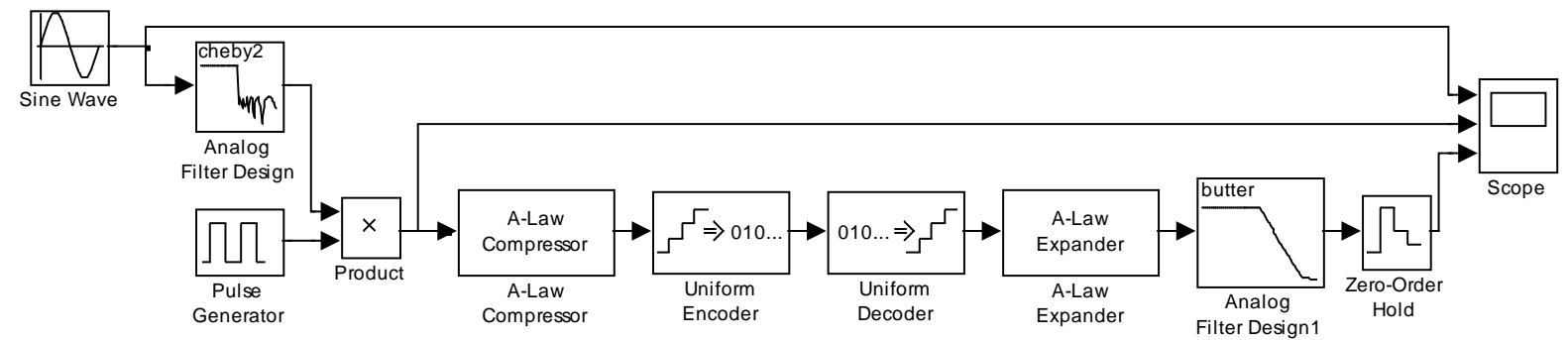

Figure 4. Pulse Amplitude Modulation \& Demodulation Model

In this example, we use signal generator to generate Sin Wave as the source, the Sin Wave is delivered to a Chebyshev II lowpass analog filter, and then multiply with the a periodic pulse, and then use A-law Compressor to quantize signal. At the receiver, we use A-law expander, Butterworth lowpass filter and Zero-Order Hold to recover the received signal. The simulation waves are shown 
in Figure 5. The first line is the original signal at the transmitter, the second line is the sampled signal and the last line is the recovered signal at the receiver.

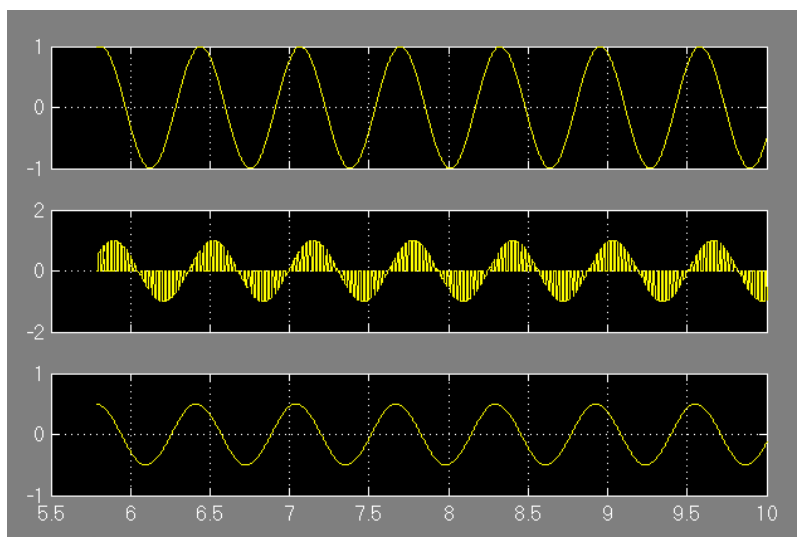

Figure 5. PAM based Communication System Simulation Results
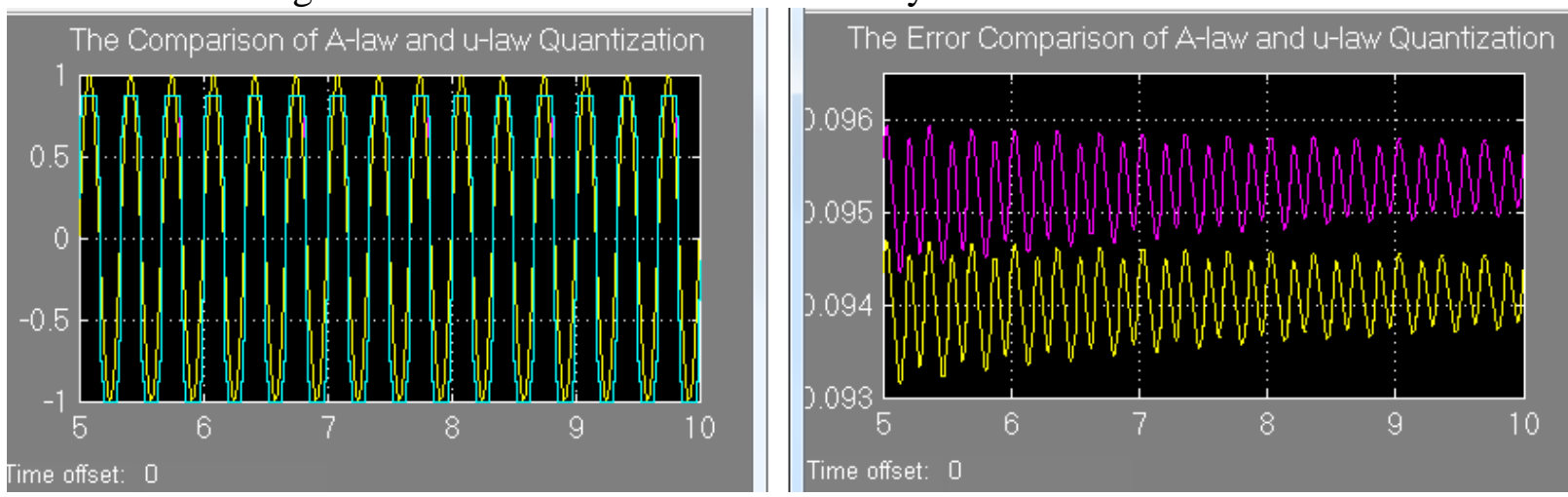

Figure 6. Wave and Error Rate Comparison of A-law and u-law Quantization

Figure 6 is the comparison of A-law and u-law quantization, and the Error Rate of these two types of quantization. From it we can see that A-law quantization has high efficiency and lower error rate.

\subsection{Amplitude Modulation/Demodulaiton Model}

AM [7] has been in use since the very earliest days of radio technology. It is most commonly used for sending signal via a radio carrier wave, and used especially as a means of broadcasting an audio signal by combining it with a radio carrier wave. In AM based communication, the amplitude of the carrier varies in accordance with the instantaneous amplitude of the modulating signal.

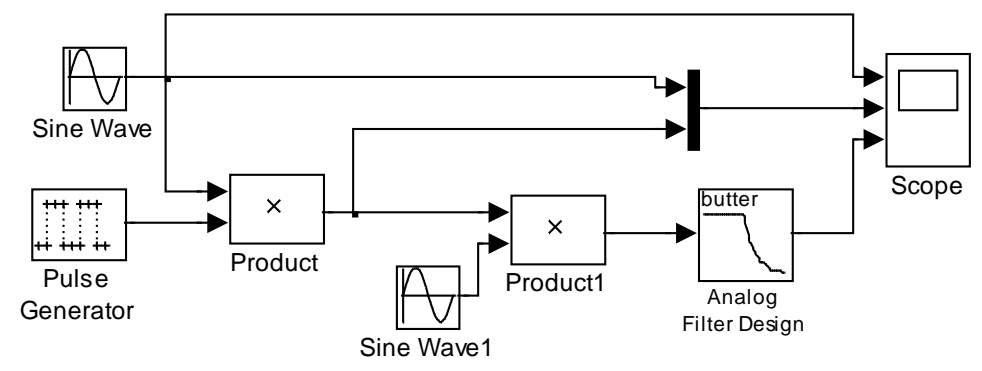

Figure 7. AM Simulation Model 


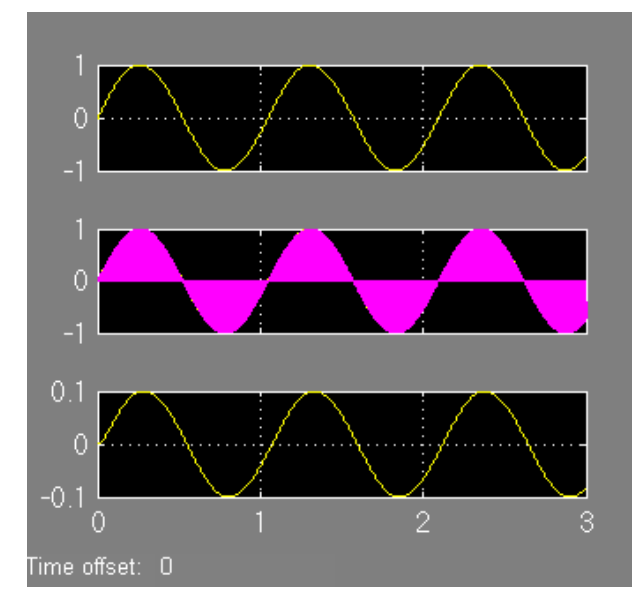

Figure 8. Waves of AM Communication System

Figure 7 is a AM communication system simulation model. There is a Sin wave generator, a pulse generator, and a multiplier at the transmitter, and at the receiver, the modulated AM signal is demodulated using coherent demodulation method. Figure 8 is the simulation results. The first line is the original signal, it is a Sin wave with the frequency of $30 \mathrm{~Hz}$, the second line is the sampled signal and the last line is the recovered signal at the transmitter.

In this experiment, students can adjust the wave frequency and even the signal waves to further development of the AM system, and students also can follow the above principle to design DSB, SSB communication system and make a comparison to make a deeper understanding of the relative knowledge.

\section{Conclusion}

Matlab/Simulink is one of the most outstanding software, which is of great significance to the reform of Communication Engineering courses, and it is also a great assistant tool for traditional education of other majors, such as electricity, information, mathematics, automation and so on.

As a useful tool for both teaching and research, Matlab/Simulink can help teachers and students to design systems according with the courses content and research direction. And it could avoid many drawback of traditional teaching and research, for example, the traditional hardware experimental platform is fixed and can not do second development.

Using Matlab/Simulink as an assistant aid in teaching, students can grasp many complicate abstract concepts in a much easier way. For example, the concept of QPSK modulation, QAM modulation and principle of synchronic control, could be vivid expressed through Matlab/Simulink visual simulation function. Students can design communication systems based on the knowledge they learn from the class and simulation, analysis the simulation results, in this way, they can get a better understanding of the knowledge and improve their design ability and cultivate their innovation capability.

\section{References}

[1] http://www.yorku.ca/jdc/Matlab/Lesson1.htm

[2] Mingyuan Xu, Yubing Shao, Communication System Modeling and Simulation Examples Analysis based on Matlab/Simulink, The press of Xi'an University of Electronic Science and Technology.

[3] https://en.wikipedia.org/wiki/Frequency_modulation.

[4] https://en.wikipedia.org/wiki/FM_broadcasting\#Stereo_FM.

[5] https://en.wikipedia.org/wiki/Pulse-amplitude_modulation.

[6] Mingyuan Xu, Yubing Shao, The application of Modern Communication based on Matlab 
Simulation, Xi'an University of Electronic Science and Technology, Press.

[7] https://en.wikipedia.org/wiki/Amplitude_modulation.

[8] Aleksei Tepljakov; Eduard Petlenkov; Emmanuel A. Gonzalez; Ivo Petras, Design of a MATLAB-based teaching tool in introductory fractional-order systems and controls, 2017 IEEE Frontiers in Education Conference (FIE)

[9] Hugo G. Espinosa; David V. Thiel, MATLAB-Based Interactive Tool for Teaching Electromagnetics, IEEE Antennas and Propagation Magazine, Year: 2017, Volume: 59, Issue: 5, Pages: 140 - 146

[10] Subhan Khan; Mujtaba Hussain Jaffery; Athar Hanif; Muhammad Rizwan Asif, Teaching Tool for a Control Systems Laboratory Using a Quadrotor as a Plant in MATLAB, IEEE Transactions on Education, Year: 2017, Volume: 60, Issue: 4, Pages: 249 - 256.

[11] Ismaila B. Tijani, Teaching fundamental concepts in robotics technology usingMATLAB toolboxes, 2016 IEEE Global Engineering Education Conference, Year: 2016, Pages: 403 - 408. 